\title{
On mixing at the core-envelope interface during classical nova outbursts
}

\author{
J. Casanova ${ }^{1}$, J. José ${ }^{1}$, E. García-Berro ${ }^{2}$, A. Calder ${ }^{3}$, and S. N. Shore ${ }^{4}$ \\ 1 Dept. Física i Enginyeria Nuclear, EUETIB, Universitat Politècnica de Catalunya, c/Comte d'Urgell 187, 08036 Barcelona, Spain, \\ \& Institut d'Estudis Espacials de Catalunya, c/Gran Capità 2-4, Ed. Nexus-201, 08034 Barcelona, Spain \\ e-mail: jordi.jose@upc.edu \\ 2 Dept. de Física Aplicada, Universitat Politècnica de Catalunya, c/Esteve Terrades 5, 08860 Castelldefels, Spain \& Institut d'Estudis \\ Espacials de Catalunya, c/Gran Capità 2-4, Ed. Nexus-201, 08034 Barcelona, Spain \\ 3 Department of Physics \& Astronomy, Stony Brook University, Stony Brook, NY 11794-3800, USA \\ 4 Dipartimento di Fisica "Enrico Fermi”, Università di Pisa and INFN, Sezione di Pisa, Largo B. Pontecorvo 3, 56127 Pisa, Italy
}

Received 1 February 2010 / Accepted 16 March 2010

\section{ABSTRACT}

\begin{abstract}
Classical novae are powered by thermonuclear runaways that occur on the white dwarf component of close binary systems. During these violent stellar events, whose energy release is only exceeded by gamma-ray bursts and supernova explosions, about $10^{-4}-10^{-5} M_{\odot}$ of material is ejected into the interstellar medium. Because of the high peak temperatures attained during the explosion, $T_{\text {peak }} \sim(1-4) \times 10^{8} \mathrm{~K}$, the ejecta are enriched in nuclear-processed material relative to solar abundances, containing significant amounts of ${ }^{13} \mathrm{C},{ }^{15} \mathrm{~N}$, and ${ }^{17} \mathrm{O}$ and traces of other isotopes. The origin of these metal enhancements observed in the ejecta is not wellknown and has puzzled theoreticians for about 40 years. In this paper, we present new 2-D simulations of mixing at the core-envelope interface. We show that Kelvin-Helmholtz instabilities can naturally lead to self-enrichment of the solar-like accreted envelopes with material from the outermost layers of the underlying white dwarf core, at levels that agree with observations.
\end{abstract}

Key words. novae, cataclysmic variables - nuclear reactions, nucleosynthesis, abundances - convection - hydrodynamics instabilities - turbulence

\section{Introduction}

The assumption of spherical symmetry in classical nova models (and in general, in stellar explosions) excludes an entire sequence of events associated with the way that a thermonuclear runaway (hereafter, TNR) initiates (presumably as a point-like ignition) and propagates. The first study of localized TNRs on white dwarfs was carried out by Shara (1982) on the basis of semianalytical models. He suggested that heat transport was too inefficient to spread a localized TNR to the entire white dwarf surface, concluding that localized, volcanic-like TNRs were likely to occur. But his analysis, based only on radiative and conductive transport, ignored the major role played by convection on the lateral thermalization of a TNR.

The importance of multidimensional effects for TNRs in thin stellar shells was revisited by Fryxell \& Woosley (1982). In the framework of nova outbursts, the authors concluded that the most likely scenario involves TNRs propagated by smallscale turbulence. On the basis of dimensional analysis and flame theory, the authors derived the velocity of the deflagration front spreading through the stellar surface, in the form $v_{\text {def }} \sim\left(h_{\mathrm{p}} v_{\text {conv }} / \tau_{\text {burn }}\right)^{1 / 2}$, where $h_{\mathrm{p}}$ is the pressure scale height, $v_{\text {conv }}$ the characteristic convective velocity, and $\tau_{\text {burn }}$ the characteristic timescale for fuel burning. Typical values for nova outbursts yield $v_{\text {def }} \sim 10^{4} \mathrm{~cm} \mathrm{~s}^{-1}$ (that is, the flame propagates halfway throughout the stellar surface in about $\sim 1.3$ days). Shear-driven mixing induced by accretion of matter possessing angular momentum was also investigated by Kutter \& Sparks (1987), but their numerical simulations failed to obtain a strong enough TNR to power a nova outburst (see Sparks \& Kutter 1987).

The first multidimensional hydrodynamic calculations of this process were performed by Shankar et al. (1992) and Shankar \& Arnett (1994). They evolved an accreting, $1.25 M_{\odot}$ white dwarf with a 1-D hydro code that was mapped into a 2 -D domain (a spherical-polar grid of $25 \times 60 \mathrm{~km}$ ). The explosive event was then followed with a 2-D version of the Eulerian code PROMETHEUS. A 12-isotope network, ranging from $\mathrm{H}$ to ${ }^{17} \mathrm{~F}$, was included to treat the energetics of the explosion. Unfortunately, the subsonic nature of the problem, coupled with the use of an explicit code (with a timestep limited by the Courant-Friedrichs-Levy condition), posed severe limitations on the study, which had to be restricted to very extreme (rare) cases, characterized by huge temperature perturbations of about $\sim 100-600 \%$, in small regions at the base of the envelope. The total computed time was only about $1 \mathrm{~s}$. The calculations revealed that instantaneous, local temperature fluctuations cause Rayleigh-Taylor instabilities. Their rapid rise and subsequent expansion (in a dynamical timescale) cools the hot material and halts the lateral spread of the burning front, suggesting that such local temperature fluctuations are not important in the initiation or early stages of the TNR. The study, therefore, favored the local volcanic-like TNRs proposed by Shara (1982).

Glasner \& Livne (1995) and Glasner et al. (1997; GLT97) revisited these early attempts using 2-D simulations performed with the code VULCAN, an arbitrarily Lagrangian Eulerian (ALE) hydrocode capable of handling both explicit and implicit steps. As in Shankar et al. (1992), a slice of the star $\left(0.1 \pi^{\mathrm{rad}}\right)$, 
in spherical-polar coordinates with reflecting boundary conditions, was adopted. The resolution near the envelope base was around $5 \times 5 \mathrm{~km}$. As before, the evolution of an accreting, $1 M_{\odot}$ $\mathrm{CO}$ white dwarf was initially followed using a 1-D hydro code (to overcome the early, computationally challenging phases of the TNR), and then mapped into a 2-D domain as soon as the temperature at the envelope base reached $T_{\mathrm{b}} \sim 10^{8} \mathrm{~K}$. As in the previous works, the 2-D runs relied on a 12-isotope network. The simulations showed a good agreement with the gross picture described by 1-D models (for instance, the critical role played by the $\beta^{+}$-unstable nuclei ${ }^{13} \mathrm{~N},{ }^{14,15} \mathrm{O}$, and ${ }^{17} \mathrm{~F}$, in the ejection stage, and consequently, the presence of large amounts of ${ }^{13} \mathrm{C},{ }^{15} \mathrm{~N}$, and ${ }^{17} \mathrm{O}$ in the ejecta). However, some remarkable differences were also identified. The TNR was initiated by an ensemble of irregular, localized eruptions at the envelope base caused by buoyancy-driven temperature fluctuations indicating that combustion proceeds in a host of many localized flames - not as a thin front - each surviving only a few seconds. Nevertheless, these authors concluded that turbulent diffusion efficiently dissipates any local burning around the core, so the fast stages of the TNR cannot be localized and the runaway must spread through the entire envelope. In contrast to 1-D models, the core-envelope interface was convectively unstable, providing a source for the metallicity enhancement of the envelope by means of a KelvinHelmholtz instability - resembling the convective overshooting proposed by Woosley (1986). Efficient dredge-up of CO material from the outermost white dwarf layers accounts for $\sim 30 \%$ metal enrichment of the envelope (the accreted envelope was assumed to be solar-like, without any pre-enrichment), in agreement with the inferred metallicites in the ejecta from $\mathrm{CO}$ novae (Gehrz et al. 1998). Finally, larger convective eddies were observed, extending up to $2 / 3$ of the envelope height with typical velocities $v_{\text {conv }} \sim 10^{7} \mathrm{~cm} \mathrm{~s}^{-1}$. Despite these differences, however, the expansion and progress of the TNR towards the outer envelope quickly became almost spherically symmetric, although the initial burning process was not.

The results of another set of 2-D simulations were published shortly afterward by Kercek et al. (1998; KHT98), which aimed to confirm the general behaviors reported by GLT97, in this case with a version of the Eulerian PROMETHEUS code. A similar domain (a box of about $1800 \times 1100 \mathrm{~km}$ ) was adopted, but using a cartesian, plane-parallel geometry to allow the use of periodic boundary conditions. Two resolution simulations were performed, one with a coarser $5 \times 5 \mathrm{~km}$ grid as in GLT97, and a second with a finer $1 \times 1 \mathrm{~km}$ grid. The calculations used the same initial model as GLT97, and produced qualitatively similar but somewhat less violent outbursts. In particular, they obtained longer TNRs with lower $T_{\text {peak }}$ and $v_{\text {ejec }}$, caused by large differences in the convective flow patterns. Whereas GLT97 found that a few, large convective eddies dominated the flow, most of the early TNR was now governed by small, very stable eddies (with $l_{\max } \sim 200 \mathrm{~km}$ ), which led to more limited dredge-up and mixing episodes. The authors attributed these discrepancies to the different geometry and, more significantly, to the boundary conditions adopted in both simulations.

The only 3-D nova simulation to date was performed by Kercek et al. (1999), adopting a computational domain of $1800 \times$ $1800 \times 1000 \mathrm{~km}$ with a resolution of $8 \times 8 \times 8 \mathrm{~km}$. It produced flow patterns that were dramatically different from those found in the 2-D simulations (much more erratic in the 3-D case), including mixing by turbulent motions occurring on very small scales (not fully resolved with the adopted resolution) and peak temperatures being achieved that were slightly lower than in the 2-D case (a consequence of the slower and more limited dredge-up of core material). The envelope attained a maximum velocity that was a factor $\sim 100$ smaller than the escape velocity and, presumably, no mass ejection (except for a possible wind mass-loss phase). In view of these results, the authors concluded that $\mathrm{CO}$ mixing must take place prior to the TNR, in contrast to the main results of GLT $97^{1}$.

In summary, two independent studies, GLT97 and KHT98, based upon the same 1-D initial model, reached nearly opposite conclusions about the strength of the runaway and its capability to power a fast nova. The origin of these differences was carefully analyzed by Glasner et al. (2005), who concluded that the early stages of the explosion, prior to the onset of the TNR when the evolution is almost quasi-static - are extremely sensitive to the outer boundary conditions (see e.g., Glasner et al. 2007, for a 2-D nova simulation initiated when the temperature at the envelope base is only $5 \times 10^{7} \mathrm{~K}$ ). Several outer boundary conditions were examined. The study showed that Lagrangian simulations, where the envelope is allowed to expand and mass is conserved, are consistent with spherically symmetric solutions. In contrast, in Eulerian schemes with a "free outflow" outer boundary condition - the choice adopted in KHT98 - the outburst can be artificially quenched.

In light of these conundrums, a reanalysis of the role of late mixing at the core-envelope interface during nova outbursts seems mandatory. To this end, we performed an independent 2-D simulation, identical to GLT97 and KHT98, with another multidimensional hydrodynamic code to investigate whether mixing can occur in an Eulerian framework with an appropriate choice of the outer boundary conditions.

\section{Models and input physics}

The 2-D simulation reported in this paper used FLASH, a parallelized, hydrodynamical, Eulerian code based on the piecewise parabolic interpolation of physical quantities for solving the hydrodynamical equations and with an adaptive mesh refinement procedure. FLASH also uses a monotonicity constraint (rather than artificial viscosity) to control oscillations near discontinuities, a feature shared with the MUSCL scheme of van Leer (1979). For consistency with GLT97 and KHT98, the same initial model was used. The model was computed by GLT97 on the basis of a 1-D, implicit hydro code, assuming accretion of solar composition matter $(Z=0.02)$ onto the surface of a $1 M_{\odot}$ $\mathrm{CO}$ white dwarf at a rate of $5 \times 10^{-9} M_{\odot} \mathrm{yr}^{-1}$. The accumulation of matter in degenerate conditions drives a temperature increase in the envelope, resulting in a superadiabatic temperature gradient and eventually convective transport. The initial model corresponds to the time when the temperature at the innermost envelope zone is $\approx 10^{8} \mathrm{~K}$. At this stage, the mass of the accreted envelope reaches $2 \times 10^{-5} M_{\odot}$. This radial profile has been mapped onto a 2-D cartesian grid of $800 \times 800 \mathrm{~km}$ and is initially relaxed to guarantee hydrostatic equilibrium. The initial computational grid comprises 112 radial layers (including the outermost part of the CO core) and 512 lateral layers. Calculations rely on the adaptive mesh refinement with a minimum resolution $1.6 \times 1.6 \mathrm{~km}$ (simulations with a finer resolution will be presented in a forthcoming publication).

\footnotetext{
1 Other multidimensional studies (Rosner et al. 2001; Alexakis et al. $2004 a, b)$ focused on the role of shear instabilities in the stratified fluids that form nova envelopes. They concluded that mixing can result from the resonant interaction between large-scale shear flows in the accreted envelope and gravity waves at the interface between the envelope and the underlying white dwarf. However, to account for significant mixing, a very high shear (with a specific velocity profile) had to be assumed.
} 

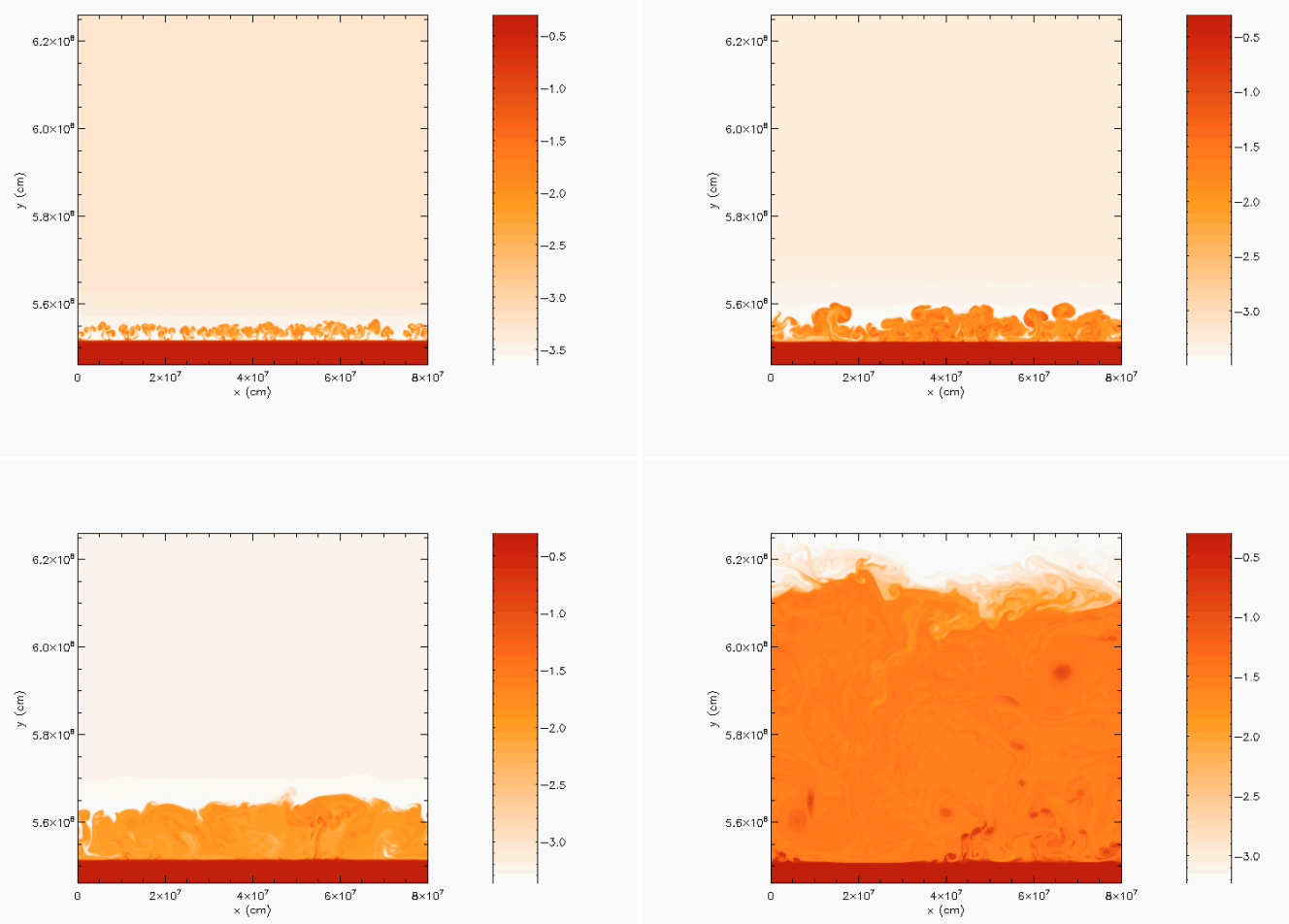

Fig. 1. Snapshots of the development of KH instabilities at $t=215 \mathrm{~s}$ (upper left panel), $235 \mathrm{~s}$ (upper right), $279 \mathrm{~s}$ (lower left), and $498 \mathrm{~s}$ (lower right), shown in terms of ${ }^{12} \mathrm{C}$ mass fraction (in logarithmic scale). The injection of core material driven by the $\mathrm{KH}$ instabilities translates into a mass-averaged abundance of CNO-nuclei in the envelope of 0.079, 0.082, 0.089, and 0.17, respectively. The mean CNO abundance at the end of the simulations reaches 0.30 , by mass.

A reduced nuclear reaction network was used to compute the energetics of the explosion: it consists of 13 isotopes $\left({ }^{1} \mathrm{H},{ }^{4} \mathrm{He}\right.$, ${ }^{12,13} \mathrm{C},{ }^{13,14,15} \mathrm{~N},{ }^{14,15,16,17} \mathrm{O}$, and ${ }^{17} \mathrm{~F}-$ as in GLT97 and KHT98 - supplemented with ${ }^{18} \mathrm{~F}$ to include the important ${ }^{17} \mathrm{O}(\mathrm{p}, \gamma){ }^{18} \mathrm{~F}$ reaction), linked through a net of 18 nuclear processes (mainly, p-captures and $\beta^{+}$-decays). Reaction rates are taken from Angulo et al. (1999) and some more recent updates (see José et al. 2006; José \& Shore 2008; and references therein).

Periodic boundary conditions were adopted at both lateral sides, while hydrostatic boundary conditions are fixed at both the bottom (reflecting) and the top (outflow) ${ }^{2}$. The set of boundary conditions are similar to those implemented in GLT97 and KHT98, but note that the outer computational grid adopted in GLT97 is Lagrangian instead of Eulerian (to follow the late expansion stages of the TNR). Finally, energy transport is included using an effective thermal diffusion coefficient that includes radiative and conductive opacities (Timmes 2000).

\section{Results}

In GLT97, significant numerical noise was present at the onset of their calculations that produced temperature fluctuations of about $10-20 \%$. We introduced (just at the initial time-step) a Gaussian temperature perturbation at the core-envelope interface of $5 \%$. For comparison, the value in KHT98 was $1 \%$. The size of the initial perturbation was $2 \mathrm{~km}$, much smaller than the contemporary depth of the accreted envelope $(\sim 800 \mathrm{~km})$. The initial perturbation produces fluctuations that move along

2 Technical details of how boundary conditions are implemented in Godunov-type codes can be found in Zingale et al. (2002). the core-envelope interface during the first seconds of the simulations. These fluctuations, in turn, spawn Kelvin-Helmholtz vortices, which clearly show up about $200 \mathrm{~s}$ later (Fig. 1), and appear to initiate a turbulent cascade. Filaments and buoyant plumes are fully resolved in these simulations. At this stage, the fluid is characterized by a large Reynolds number, with a characteristic eddy length of $50 \mathrm{~km}$, fluid velocities of $10^{5}-10^{6} \mathrm{~cm} \mathrm{~s}^{-1}$, and a dynamic viscosity of $10^{4} \mathrm{P}$. These Kelvin-Helmholtz (KH) instabilities transport unburnt $\mathrm{CO}$-rich material from the outmost layers of the white dwarf core and inject it into the envelope. The characteristic eddy turnover time is $l_{\text {conv }} / v_{\text {conv }} \sim 10 \mathrm{~s}$.

As the $\mathrm{KH}$ vortices grow in size, more $\mathrm{CO}$-rich material is transferred into the envelope. Convection becomes more turbulent. The initially small convective eddies merge into huge shells (Fig. 2), as seen also in GLT97. At this stage, the nuclear energy generation rate reaches $10^{15} \mathrm{erg} \mathrm{g}^{-1} \mathrm{~s}^{-1}$, while the characteristic burning timescale decreases to $\sim 5 \mathrm{~s}$. The convective filaments continue growing in size and progressively occupy the whole envelope length. Although not resolved in these simulations, and in contrast to the 3-D case, the conservation of vorticity in 2-D forces the largest eddies to grow in an inverse vorticity cascade, while energy flows to the viscous scale with a distribution that deviates from the Kolmogorov spectrum (see e.g., Lesieur et al. 2001; Shore 2007). At this time, the temperature at the envelope base reaches $\sim 2 \times 10^{8} \mathrm{~K}$, at fluid velocities of $10^{8} \mathrm{~cm} \mathrm{~s}^{-1}$ (of the order of the escape velocity, characteristic of the dynamic phases of the explosion), and a nuclear energy generation rate of $10^{16} \mathrm{erg} \mathrm{g}^{-1} \mathrm{~s}^{-1}$. The convective turnover time is now $\sim 5 \mathrm{~s}$. The mean CNO abundance in the envelope has increased to 0.30 , a value that agrees well with both the previous simulations by GLT97 and the mean metallicities inferred from observations of 

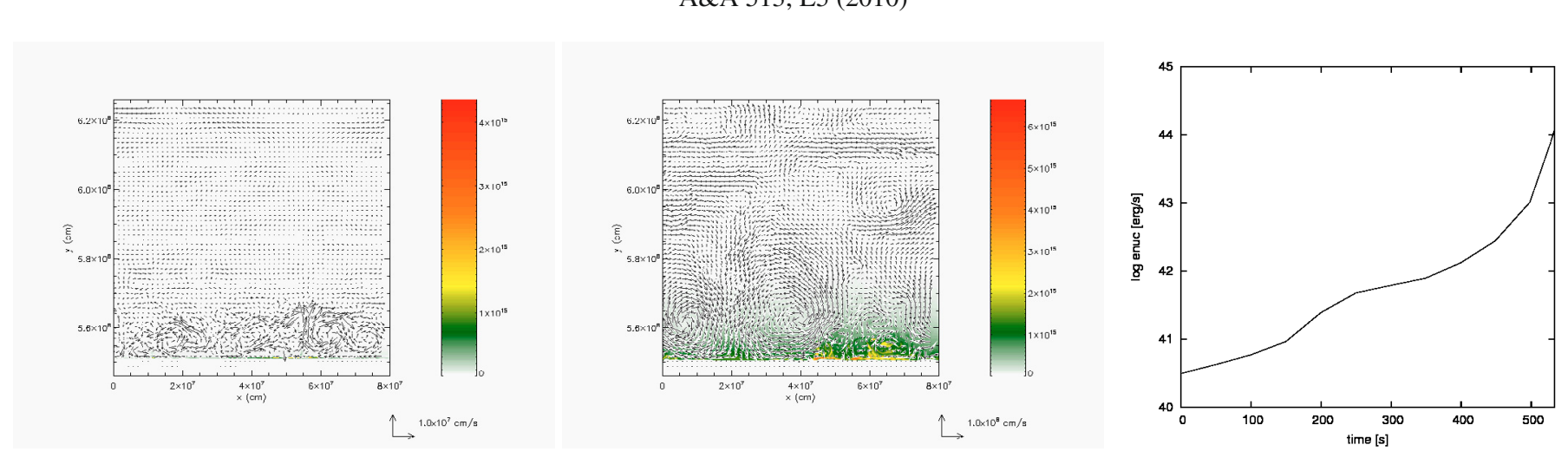

Fig. 2. Left and central panels: same as Fig. 1, but for the velocity fields at $\mathrm{t}=279 \mathrm{~s}$ (left) and $498 \mathrm{~s}$ (right), superimposed on a plot of the nuclear energy generation rate (in ergs $\mathrm{g}^{-1} \mathrm{~cm}^{-1}$ ). Right panel: Time evolution of the overall nuclear burning rate.

the ejecta in non-neon (CO) novae (see José \& Shore 2008). At this stage, since the outer envelope layers had started to escape the computational (Eulerian) domain, simulations were stopped.

Our 2-D simulations, in agreement with the results reported in GLT97, show that the progress and extension of the TNR throughout the envelope occurs with almost spherical symmetry, even though the structure of the ignition is not. This explains the success of 1-D models in reproducing the gross observational properties (light curves, velocities of the ejecta, nucleosynthesis) of nova explosions (Starrfield et al. 1998, 2009; Kovetz \& Prialnik 1997; Yaron et al. 2005; José \& Hernanz 1998).

\section{Discussion and conclusions}

We have analyzed the possible self-enrichment of the solarcomposition accreted envelope with material from the underlying white dwarf during nova outbursts in a multidimensional framework. We have found that a shear flow at the core-envelope interface (which unlike the spherically symmetric case, does not behave like a rigid wall) drives mixing through $\mathrm{KH}$ instabilities. Large convective eddies develop close to the core-envelope interface, of a size comparable to the height of the envelope (similar to the pressure scale height in 1-D simulations), mixing $\mathrm{CO}$-rich material from the outermost layers of the underlying white dwarf into the accreted envelope. The metallicity enrichment achieved in the envelope, $Z \sim 0.30$, is in agreement with observations of CO nova ejecta. Our 2-D simulations also show that even for a point-like TNR ignition, the expansion and progress of the runaway is almost spherically symmetric for nova conditions. We note that the adopted resolution as well as the size, intensity, and location of the initial perturbation have a very limited impact on the results, principally affecting the timescale for the onset of the KH instability but not the final, mean metallicity. Details will be extensively discussed in a forthcoming publication. Our results agree with earlier 2-D hydrodynamic simulations (GLT97) and solve the controversy raised by another 2-D study (KHT98) that questioned the efficiency of this mixing mechanism, and hence the corresponding strength of the runaway and its capability to power a fast nova outburst.
Acknowledgements. We greatly appreciate comments and suggestions from D. García-Senz, J. Isern, A. Parikh, and M. Zingale on an early version of this manuscript. This work has been partially supported by the Spanish MEC grants AYA2007-66256 and AYA2008-04211-C02-01, and by the E.U. FEDER funds. We also acknowledge the Barcelona Supercomputing Center for a generous allocation of time at the Mare Nostrum supercomputer.

\section{References}

Alexakis, A., Calder, A. C., Heger, A., et al. 2004a, ApJ, 602, 931

Alexakis, A., Calder, A. C., Dursi, L. J., et al. 2004b, Phys. Fluids, 16, 3256 Angulo, C., Arnould, M., Rayet, M., et al. 1999, Nucl. Phys. A, 656, 3 Calder, A., Alexakis, A., Dursi, L. J., et al. 2002, in Classical Nova Explosions, ed. M. Hernanz, \& J. José (New York: AIP), AIP Conf. Proc., 637, 134 Fryxell, B. A., \& Woosley, S. E. 1982, ApJ, 261, 332

Gehrz, R. D., Truran, J. W., Williams, R. E., \& Starrfield, S. 1998, PASP, 110, 3 Glasner, S. A., \& Livne, E. 1995, ApJ, 445, L149

Glasner, S. A., Livne, E., \& Truran, J. W. 1997, ApJ, 475, 754 (GLT97)

Glasner, S. A., Livne, E., \& Truran, J. W. 2005, ApJ, 625, 347

Glasner, S. A., Livne, E., \& Truran, J. W. 2007, ApJ, 665, 1321

José, J., \& Hernanz, M. 1998, ApJ, 494, 680

José, J., \& Shore, S. 2008, in Classical Novae, ed. M. F. Bode, \& A. Evans (Cambridge: Cambridge Univ. Press), 121

José, J., Hernanz, M., \& Iliadis, C. 2006, Nucl. Phys. A, 777, 550

Kercek, A., Hillebrandt, W., \& Truran, J. W. 1998, A\&A, 337, 379 (KHT98)

Kercek, A., Hillebrandt, W., \& Truran, J. W. 1999, A\&A, 345, 831

Kovetz, A., \& Prialnik, D. 1997, ApJ, 477, 356

Kutter, G. S., \& Sparks, W. M. 1987, ApJ, 321, 386

Lesieur, M., Yaglom, A., \& David, F. (eds.) 2001, New Trends in Turbulence, Les Houches 2000 Summer School, vol. 74 (Berlin: Springer-Verlag; Les Ulis: EDP Sciences)

Rosner, R., Alexakis, A., Young, Y., Truran, J. W., \& Hillebrandt, W. 2001, ApJ, 562, L177

Shankar, A., \& Arnett, D. 1994, ApJ, 433, 216

Shankar, A., Arnett, D., \& Fryxell, B. A. 1992, ApJ, 394, L13

Shara, M. M. 1982, ApJ, 261, 649

Shore, S. N. 2007, Astrophysical Hydrodynamics (Wiley: Darmstadt)

Sparks, W. M., \& Kutter, G. S. 1987, ApJ, 321, 394

Starrfield, S., Truran, J. W., Wiescher, M., \& Sparks, W. 1998, MNRAS, 296, 502

Starrfield, S., Iliadis, C., Hix, W. R., Timmes, F. X., \& Sparks, W. M. 2009, ApJ, 692,1532

Timmes, F. X. 2000, ApJ, 528, 913

van Leer, B. 1979, J. Comput. Phys., 32, 101

Woosley, S. E. 1986, in Nucleosynthesis and Chemical Evolution, ed. B. Hauck,

A. Maeder, \& G. Meynet (Geneva Observatory: Sauverny), 1

Yaron, O., Prialnik, D., Shara, M. M., \& Kovetz, A. 2005, ApJ, 623, 398

Zingale, M., Dursi, L. J., ZuHone, J., et al. 2002, ApJS, 143, 539 